Research Article

\title{
Microchip Nd:YAG and Nd:YVO 4 Lasers Pumped by VHG Wavelength-Stabilized Laser Diode
}

\author{
T. Saiki (iD) and A. Tatebayashi \\ Kansai University, Department of Electrical, Electronic and Information Engineering, Faculty of Engineering Science, \\ 3-3-35 Yamate-Cho, Suita, Osaka 564-8680, Japan \\ Correspondence should be addressed to T. Saiki; tsaiki@kansai-u.ac.jp
}

Received 31 January 2020; Revised 12 May 2020; Accepted 3 July 2020; Published 1 August 2020

Academic Editor: Paramasivam Senthilkumaran

Copyright (c) 2020 T. Saiki and A. Tatebayashi. This is an open access article distributed under the Creative Commons Attribution License, which permits unrestricted use, distribution, and reproduction in any medium, provided the original work is properly cited.

\begin{abstract}
We adopted a single-mode, single-wavelength volume holographic grating (VHG) wavelength-stabilized wavelength laser diode (LD) as a pumping LD for an end-pumped microchip Nd:YAG and $\mathrm{Nd}: \mathrm{YVO}_{4}$ lasers we developed during CW and pulse operations. Higher optical-optical and slope efficiencies during CW operation have been obtained than when using a VHG LD experimentally. Output laser power is insensitive to the temperature of the LD when using a wavelength-stabilized LD and can remain stable and almost constant until the temperature of $\mathrm{LD}$ increases up to $40^{\circ} \mathrm{C}$. The improved optical-optical conversion efficiency of $58 \%$ for the $\mathrm{Nd}: \mathrm{YVO}_{4}$ laser has been obtained and calculated the output laser power during CW operation and compared it with the experimental results. We found that the output laser power of the $\mathrm{Nd}_{\mathrm{YVO}} \mathrm{Y}_{4}$ laser using the VHG wavelength-stabilized LD was more than twice as high as that using an LD without VHG. When the ambient temperature increases, the difference in output laser power should be large. In the future, a low-cost end-pumped microchip laser that does not require a temperature control should be developed.
\end{abstract}

\section{Introduction}

Solid-state lasers are compact and efficient and have high beam quality, and the application of laser diode- (LD-) pumped lasers to the industrial field has expanded. Various end-pump microchip solid-state lasers under CW and pulse operations have been developed [1-16]. Current Nd:YAG/ Cr:YAG microchip lasers realize over $1 \mathrm{~mJ}$ with over $1 \mathrm{MW}$ of peak power [4]. A microchip solid-state laser can generate a high gain with low loss in a small volume, can easily obtain high beam quality and a short pulse, and is compact, comparable to an LD. Direct processing using an LD is possible; however, an LD is extremely difficult to operate with a pulse duration in ns because this would limit the lifetime of an LD module. End-pumped microchip lasers can easily generate ns-laser pulses with high peak power. Neodymium-doped yttrium aluminum garnet (Nd:YAG) crystals have been used as laser media for microchip lasers; however, the absorption bandwidth for the pump light is as narrow as $2.5 \mathrm{~nm}$ [17], and when the temperature of the $\mathrm{LD}$ increases, the peak wavelength of the output light shifts to the longer wavelength side, so LD temperature should be controlled. Thus, the cost of laser systems increases.

The possibility of a laser using a neodymium-doped yttrium orthovanadate $\left(\mathrm{Nd}: \mathrm{YVO}_{4}\right)$ crystal was reported in 1966 [2]. An Nd:YVO ${ }_{4}$ crystal has a stimulated emission cross section, which is three times higher than that of an $\mathrm{Nd}$ : YAG crystal, and has a seven times higher absorption coefficient at $\pi$-polarization. Higher laser power can be achieved with an $\mathrm{Nd}: \mathrm{YVO}_{4}$ crystal during $\mathrm{CW}$ operation than with an Nd:YAG crystal. However, the laser output fluctuates due to the difference in absorbed power of the pump light at low and high temperatures due to the shift in the peak wavelength of the $\mathrm{LD}$, which is not the case when using an Nd:YAG crystal.

Research has been conducted to stabilize the output frequency by using the feedback of the partial output light of an LD to semiconductors $[18,19]$. We developed an end- 
pumped $\mathrm{Nd}: \mathrm{YVO}_{4}$ laser that uses a volume holographic grating (VHG) wavelength-stabilized narrow band LD for pumping. The narrowband LD is important for laser systems because it increases the amount of absorbed light and fixes the central peak wavelength for the LD temperature. Previous studies have reported on Tm:YAG and Yb:YAG lasers pumped using a VHG-stabilized narrow band LD [20, 21], and experiments for generating repetitive pulses by Q-switching and during CW operation were conducted. Output laser power was compared with that using a conventional LD without VHG. The temporal stability of the output laser power was also investigated.

\section{Calculation of Output Laser Power}

The CW output laser power for a microchip laser is calculated as follows [22]:

$$
P_{\text {out }}=\left[\frac{g_{0}}{L}-1\right] \cdot \frac{T}{L} \cdot P_{\text {sat }} \cdot A,
$$

where $A_{s}$ is the effective pumping cross section, $L$ is the loss of light in the laser cavity, $T$ is $1-R_{2}$, where $R_{2}$ is the reflectivity of the output mirror, $P_{\text {sat }}$ is the total fluorescence power in the laser cavity, and $A$ is the coupling ratio of the initial fluorescence to the cavity. The large gain $G$, small signal gain coefficient $g_{0} l$, and inversion population of the laser medium $N_{\text {inv }}$ are calculated as

$$
\begin{aligned}
G & =\exp \left(g_{0} l\right)=\exp \left(\sigma_{L} N_{\text {inv }}\right), \\
N_{\text {inv }} & =\Delta N \cdot \tau_{R},
\end{aligned}
$$

where $\Delta N$ is the pump rate per unit volume and $\tau_{R}$ is the fluorescence lifetime of the laser medium. The population density of $\mathrm{Nd}$ ions at the lower level for $\mathrm{Nd}$ :YAG and $\mathrm{Nd}$ : $\mathrm{YVO}_{4}$ is omitted because the temperatures of the laser materials are below $40^{\circ} \mathrm{C}$. The $\Delta N$ is calculated as

$$
\Delta N=\frac{\eta_{q} \cdot \eta_{a} \cdot P_{\text {in }}}{A_{s} \cdot h\left(C / \lambda_{P}\right)},
$$

where $P_{\text {in }}$ is the pump power of the LD light, $\lambda_{p}$ is the pump wavelength, $\eta_{q}$ is the emission efficiency of the laser material, and $\eta_{a}$ is the absorption rate of the pumping light. The average output laser power of a high repetitive pulse laser is calculated as

$$
P_{\text {out }}=\Delta E_{\text {out }} f \text {, }
$$

where $\Delta P$ is the energy of the single laser pulse and $f$ is the repetition rate.

The Nd:YAG parameters for calculating the output laser power are described below. We used equations (1)-(3) to calculate the CW output laser power of the $\mathrm{Nd}$ :YAG and $\mathrm{Nd}$ : $\mathrm{YVO}_{4}$ lasers. The stimulated emission cross section $\sigma_{L}$ of $\mathrm{Nd}$ : YAG and Nd:YVO $\mathrm{NO}_{4}$ crystals was set to $2.8 \times 10^{-19} \mathrm{~cm}^{2}$ and $1.6 \times 10^{-18} \mathrm{~cm}^{2}$, respectively, and $\tau_{R}$ was set to 230 and $100 \mu \mathrm{s}$, respectively [17].

The focused spot diameter of LD light on the laser materials was assumed to be $230 \mu \mathrm{m}$ for an LD without VHG and $160 \mu \mathrm{m}$ in the case of the VHG LD. Thus, $A_{s}$ was set to 0.0004 and $0.00020 \mathrm{~cm}^{2}$ when using the LD without VHG and VHG LD, respectively. The lengths of the Nd:YAG and $\mathrm{Nd}: \mathrm{YVO}_{4}$ crystal rods were set to 7 and $1 \mathrm{~mm}$, respectively. The optical loss of the laser material at the laser wavelength was ignored because the thickness of the laser materials was thin. The transmittance of the pumping light in the HR coating was set to $100 \%$. The $\eta_{q}$ of both laser materials (including optical loss) with respect to the excitation light was set to $90 \%$.

\section{Experimental Setup}

The experimental setup for the two microchip lasers is shown in Figure 1. The lasers contain either an Nd:YAG crystal (size: $3 \mathrm{~mm} \Phi, 7 \mathrm{~mm}$ thick, CASTECH) or $\mathrm{Nd} \mathrm{YVO}_{4}$ crystal (size: $3 \mathrm{~mm} \Phi, 1 \mathrm{~mm}$ thick, a-cut, CASTECH). We used an HR coating of $1064 \mathrm{~nm}$ and an AR coating of $809 \mathrm{~nm}$ on the pumping sides of the crystals. The other sides had an AR coating of $1064 \mathrm{~nm}$. For the CW laser operation, the cavity length was set to $10 \mathrm{~mm}$, and the reflectivity of the output coupling mirror was $90 \%$. Two types of LDs were used as pumping light sources: an LD without VHG (single-mode onechip, LD 808-SE500, Thorlabs) and an LD (single-mode onechip, LD 808-SEV500, Thorlabs) with VHG (hereafter, VHG LD) for partial LD optical feedback to fix the output wavelength. The peak wavelengths of the output light from the two LDs at $25^{\circ} \mathrm{C}$ were 808.5 and $808.3 \mathrm{~nm}$, respectively. The divergence angles at these peak wavelengths of the output light for the LD without VHG were $14^{\circ}$ and $7^{\circ}$ and those for the VHG LD was $14^{\circ}$ and $8^{\circ}$. The performance of the focused LD light for the VHG LD was better than that of the LD without VHG because the spatial distribution of the LD light for the VHG LD was concentrated at the center. The bandwidth of the emission spectrum at $808 \mathrm{~nm}$ was $2.5 \mathrm{~nm}$ (full width at half maximum (FWHM)) for the LD without VHG and $0.1 \mathrm{~nm}$ (FWHM) for the VHG LD. The amount of spectral peak shift for the VHG LD was $0.1 \mathrm{~nm}$ with a temperature increase of $5^{\circ} \mathrm{C}$. For the LD without VHG, the amount of peak shift was $1.0 \mathrm{~nm}$ with the same temperature increase. Since the VHG LD had a WHG near the semiconductor for returning the output LD light, the spatial pattern of the LD light in the near field was partially missing, but this does not affect the focusing pattern of the microchip laser.

The LD was set tightly in a copper holder. A temperature-control device with a Peltier cooler and electronic controller (TED200C, Thorlabs) was used to control the LD temperature.

A thermistor for the temperature measurement was installed outside the LD can. The output light from the LD entered once through a single lens with AR coating $(f=6.24 \mathrm{~mm})$ and then collected in front of the laser media. The Nd:YAG crystal was fixed in a heatsink copper folder $\left(14 \times 14 \mathrm{~mm}^{2}, 10 \mathrm{mmt}\right)$ to exhaust the generated heat. The $\mathrm{Nd}: \mathrm{YVO}_{4}$ crystal was fixed on a heatsink copper plate $\left(14 \times 14 \mathrm{~mm}^{2}, 0.5 \mathrm{mmt}\right)$ because of its thinness.

$\mathrm{A} \mathrm{Cr}^{4+}$ :YAG crystal with a diameter of $10 \mathrm{~mm}$ was set near the laser materials, as shown in Figure 1, for generating repetitive pulses by Q-switching. The initial transmittance of the $\mathrm{Cr}^{4+}: \mathrm{YAG}$ crystal was $90 \%$. A bandpass filter at the laser wavelength was used to measure the output laser power, which was normalized. 


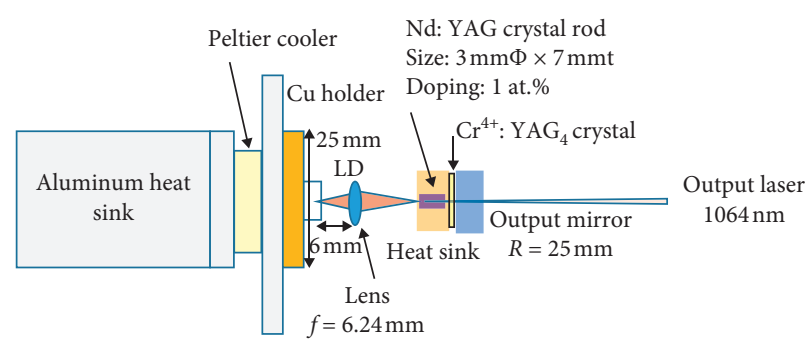

(a)

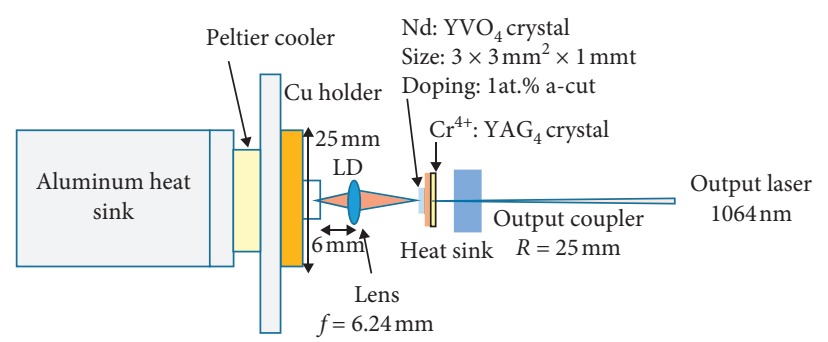

(b)

Figure 1: Experimental setup for (a) Nd:YAG and (b) Nd:YVO lasers.

\section{Experimental Results}

The experimental results for generating a CW laser are shown in Figure 2. The dots are the experimental results, and the solid lines are results calculated using equations (1)-(3). The spatial beam profiles of the microchip lasers were quasiGaussian. The room temperature was $23^{\circ} \mathrm{C}$.

For the Nd:YAG laser using the LD without VHG, the threshold for the pump power was $70 \mathrm{~mW}$, slope efficiency was $40 \%$, and maximum output conversion efficiency was $33 \%$, as shown in Figure 2. The maximum output laser power was $160 \mathrm{~mW}$. Generated heat in the semiconductor chip of a can-type LD cannot be exhausted when the output power of the LD light is high. As the temperature of the LD increases, the peak wavelength of the LD light shifts to the longer wavelength side. Thus, the output of laser power is saturated when the pump power increased to around $400 \mathrm{~mW}$.

In the range of pump power (below $370-380 \mathrm{~mW}$ ) where the thermal shift of the LD without VHG shows good superposition with the absorption line of $\mathrm{Nd}$, the slope efficiency is higher without LD stabilization. This is because the peak wavelength of the VHG LD light is a little shorter than that of the absorption band for the Nd:YAG. The peak wavelength of the output light for the LD without VHG is almost the same as that of the absorption band for the $\mathrm{Nd}$ : YAG.

The threshold was also $60 \mathrm{~mW}$ for the Nd:YAG laser using the VHG LD, but the slope efficiency was $43 \%$ and maximum output conversion efficiency was $37 \%$. The conversion efficiency and slope efficiency increased slightly. The maximum output laser power was $200 \mathrm{~mW}$.

The peak wavelength of the LD light shifts to the longer side as the temperature of LD increases. Thus, increasing the absorbed power of the LD light resulted in increasing the output laser power slightly and nonlinearly.

For the $\mathrm{Nd}: \mathrm{YVO}_{4}$ laser using LD without VHG, the threshold for the pump power was $20 \mathrm{~mW}$, slope efficiency was $47 \%$, and conversion efficiency at the maximum output was $36 \%$, as shown in Figure 3. The maximum output laser power was $160 \mathrm{~mW}$. For this laser using the VHG LD, the threshold was $10 \mathrm{~mW}$, slope efficiency was $56 \%$, and maximum output conversion efficiency was $54 \%$, as shown in Figure 3. These results indicate that the conversion and slope efficiencies improved using the VHG LD. The obtained maximum output laser power was $290 \mathrm{~mW}$. When the pump power is $600 \mathrm{~mW}$, the output laser power should reach
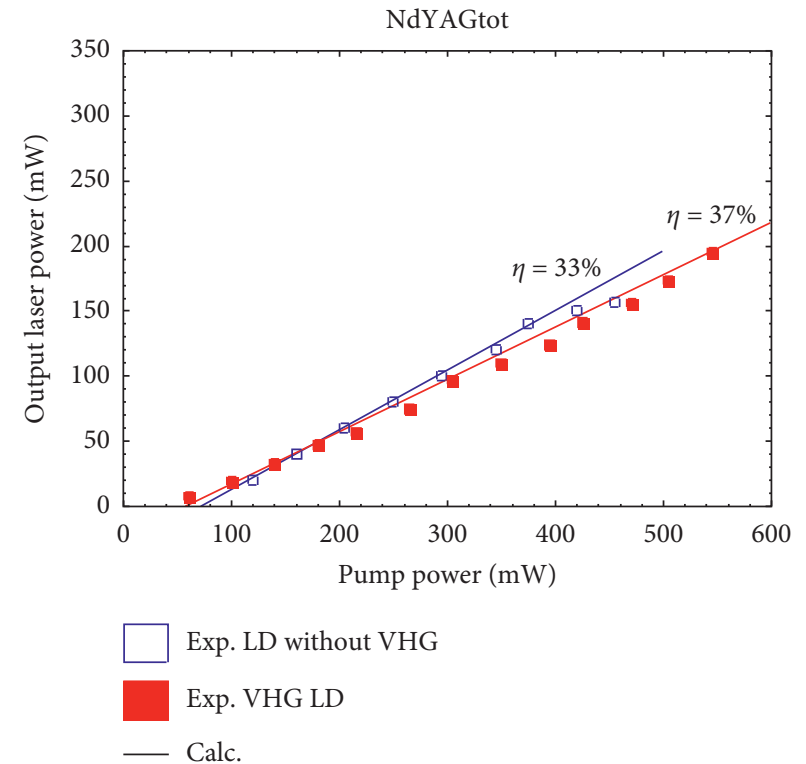

FIgURE 2: Output laser power of Nd:YAG laser during CW operation.

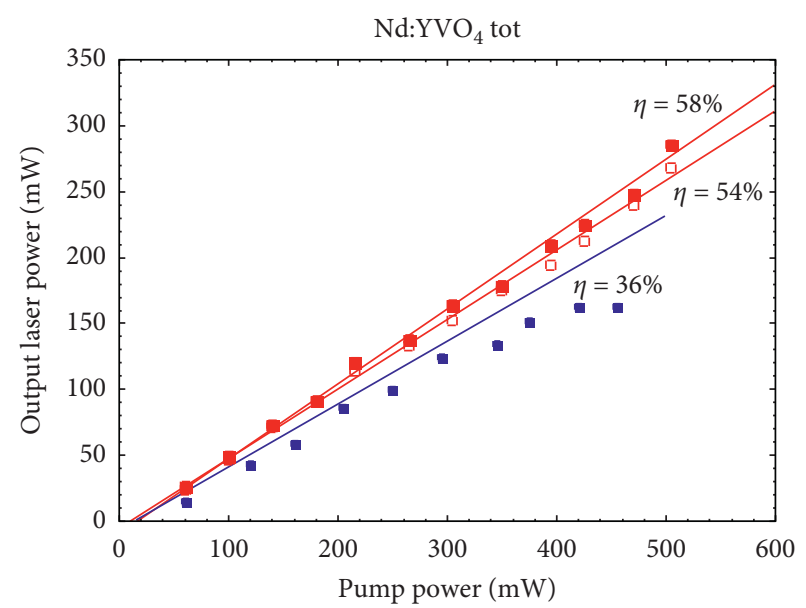

Exp. LD without VHG
Exp. VHG LD $L=10 \mathrm{~mm}$
Exp. VHG LD $L=5 \mathrm{~mm}$
Calc.

FIgURE 3: Output laser power of $\mathrm{Nd}_{\mathrm{YVO}} \mathrm{VV}_{4}$ laser during $\mathrm{CW}$ operation. 
$320 \mathrm{~mW}$. The maximum output laser power of the $\mathrm{Nd}: \mathrm{YVO}_{4}$ laser using the VHG LD was twice as high as that of it using the LD without VHG.

When we changed the resonator length for the Nd: $\mathrm{YVO}_{4}$ laser using the VGH LD to $5 \mathrm{~mm}$, the threshold was $20 \mathrm{~mW}$, slope efficiency was $59 \%$, and maximum output conversion efficiency was 58\%, as shown in Figure 3.

Normally, the output laser power of a four-level laser oscillator (such as the Nd:YAG laser) is calculated to change almost linearly with the pump power. However, when the pumping power was high in the experiment, the temperature of the laser medium increased and the output was saturated. When the pump power was about $500 \mathrm{~mW}$ in this experiment, the temperature of the laser medium was low, so the above does not apply. The difference in laser power for $L=5$ and $10 \mathrm{~mm}$ is the use of pumped volume.

The conversion and slope efficiencies increased using the VHG LD, and the obtained maximum output laser power was $290 \mathrm{~mW}$. When using the VHG LD, the threshold decreased by $50 \%$, and the slope efficiency and output power also increased. This is probably because the bandwidth of the output light from the LD narrowed, spatial mode improved, the divergence angle of the LD light decreased, and the focusing diameter of the pumping LD light decreased.

Both LD output power characteristics used in this experiment do not change with an increase in temperature and show only a slight decrease from $25^{\circ} \mathrm{C}$ to $35^{\circ} \mathrm{C}$ at the output power of $500 \mathrm{~mW}$. Even if the temperature increases $10^{\circ} \mathrm{C}$, the output power will not decrease significantly for the LD current. Exhausting the heat of the LD cannot catch up and the chip temperature increases to around $30^{\circ} \mathrm{C}$. The peak wavelength of the LD light shifts to a long wavelength and moves away from the peaks of the absorption band for the $\mathrm{Nd}$ :YAG and $\mathrm{Nd}: \mathrm{YVO}_{4}$, so the laser output decreases. When a VHG LD is used, the output power increases linearly with the pump power. This also shows that the pump power does not drop as a function of the LD current.

The calculated results of the optimum coupling reflectivity of the output mirror and conversion efficiency are shown in Figure 4. In this experiment, we set the reflectivity of the output mirror to $90 \%$. We conducted a computer analysis to investigate whether the coupling was optimal. A cavity length of $10 \mathrm{~mm}$ and the VHG LD were assumed. The pump power was set to $500 \mathrm{~mW}$ and the absorption rate was assumed maintained at $100 \%$ up to $35^{\circ} \mathrm{C}$. for the Nd:YAG laser, the optimum output mirror reflectivity was $90 \%$. In contrast, it was just $80 \%$ for the $\mathrm{Nd}: \mathrm{YVO}_{4}$ laser. The output was $295 \mathrm{~mW}$, and the conversion efficiency of $60 \%$ was expected from the calculation results. The output laser power of the $\mathrm{Nd}: \mathrm{YVO}_{4}$ laser was twice as high as that of the $\mathrm{Nd}$ : YAG laser.

The experimental results for the stability of laser power with respect to LD temperature are shown in Figure 5, where $I$ is LD current. Temperatures below $23^{\circ} \mathrm{C}$ could not be set due to the problem of the cooling system. For the Nd:YAG laser using the LD without VHG, the output laser power was $45 \mathrm{~mW}$ at $24^{\circ} \mathrm{C}$, as shown in Figure 5(a), and the pump power was $150 \mathrm{~mW}$. When the temperature increased to $35^{\circ} \mathrm{C}$, this power gradually decreased to $19 \mathrm{~mW}$. For this laser using the VHG, the output laser power was 12 and $35 \mathrm{~mW}$ at $23^{\circ} \mathrm{C}$, as shown in Figure $5(\mathrm{a})$, and the pump power was $100 \mathrm{~mW}$. When the temperature increased to $39^{\circ} \mathrm{C}$, this power was kept constant. For the $\mathrm{Nd}: \mathrm{YVO}_{4}$ laser using the LD without VHG, the output laser power was $47 \mathrm{~mW}$ at $23^{\circ} \mathrm{C}$, and the pump power was $72 \mathrm{~mW}$, as shown in Figure 5(b). When the temperature increased to $35^{\circ} \mathrm{C}$, this power gradually decreased to $35 \mathrm{~mW}$. For this laser using the VHG LD, the output laser power was $45 \mathrm{~mW}$ at $24^{\circ} \mathrm{C}$, and the pump power was $150 \mathrm{~mW}$. When the temperature increased to $39^{\circ} \mathrm{C}$, this power was kept constant, as shown in Figure 5(b).

The calculated absorption rate of laser materials as a function of the LD temperature obtained from the experimental results is shown in Figure 6. In the case of both $\mathrm{Nd}$ : YAG and Nd: $\mathrm{YVO}_{4}$, the calculated the absorption rate of the absorbed pump power was normalized by the maximum absorbed pump power. For the Nd:YAG laser using the LD without VHG, we found that the absorption rate decreased by $30 \%$ compared to that at $24^{\circ} \mathrm{C}$ when the temperature increased to $35^{\circ} \mathrm{C}$. For this laser using the VHG LD, when the temperature increased to $35^{\circ} \mathrm{C}$, the absorption rate decreased only $5 \%$ compared to that at $24^{\circ} \mathrm{C}$. For the Nd:YVO 4 laser using the LD without VHG, the absorption rate decreased by $20 \%$ when the temperature increased to $35^{\circ} \mathrm{C}$. For this laser using the VHG LD, the absorption rate did not decrease up to $35^{\circ} \mathrm{C}$. The output laser power was maintained owing to the broad effective absorption bandwidth at the pumping wavelengths of the Nd:YAG and Nd:YVO ${ }_{4}$ crystals, which are 2.5 and $15.7 \mathrm{~nm}$, respectively [17]. The absorption rate of the pump power for both laser materials could be maintained when the $\mathrm{LD}$ temperature increased to $35^{\circ} \mathrm{C}$ when using the VHG LD.

The calculated results of the $g_{0} l$ and $G$ in a small signal are shown in Figure 7. We found that $\mathrm{Nd}: \mathrm{YVO}_{4}$ had a gain coefficient 4.7 times higher than that of Nd:YAG. This is presumably because the absorption area of the focused LD light for $\mathrm{Nd}: \mathrm{YVO}_{4}$ was small. The multiplication of $\sigma_{L}$ and $\tau_{R}$ resulted in a large difference in the gain coefficient. It is clear that the Nd:YAG and Nd:YVO ${ }_{4}$ lasers can maintain $G$ even at an LD temperature of $35^{\circ} \mathrm{C}$. Particularly for the $\mathrm{Nd}: \mathrm{YVO}_{4}$ laser, a high $G$ can be maintained using the VHG LD.

In fact, the peak wavelength of the LD light shifts due to increasing the temperature of the LD. However, since the absorption band was wide for the $\mathrm{Nd}: \mathrm{YVO}_{4}$, the output could be maintained against the temperature change of the LD. In addition, the Nd:YAG had a narrow absorption band and thus exhibited a slight decrease in laser output. However, it was shown that the VHG LD is effective for maintaining the output laser power even at high temperatures when using the Nd:YAG.

The experimental results for pulse laser generation are shown in Figure 8. The spatial beam profiles of the microchip lasers were a quasi-Gaussian type.

For the Nd:YAG laser using the LD without VHG, the threshold for pumping power was $160 \mathrm{~mW}$, slope efficiency was $18 \%$, and maximum output conversion efficiency was 


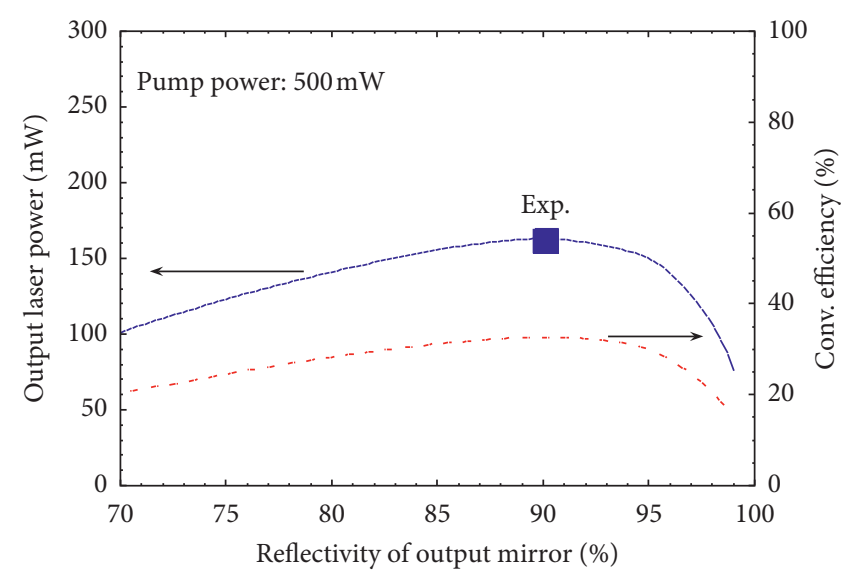

(a)

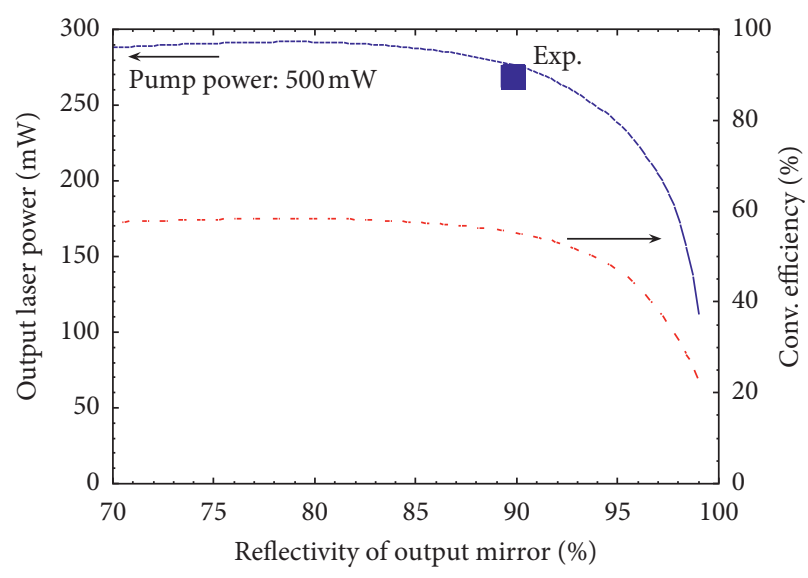

(b)

Figure 4: Calculated output laser power and conversion efficiency for the reflectivity of output mirror: (a) Nd:YAG and (b) Nd:YVO 4 .

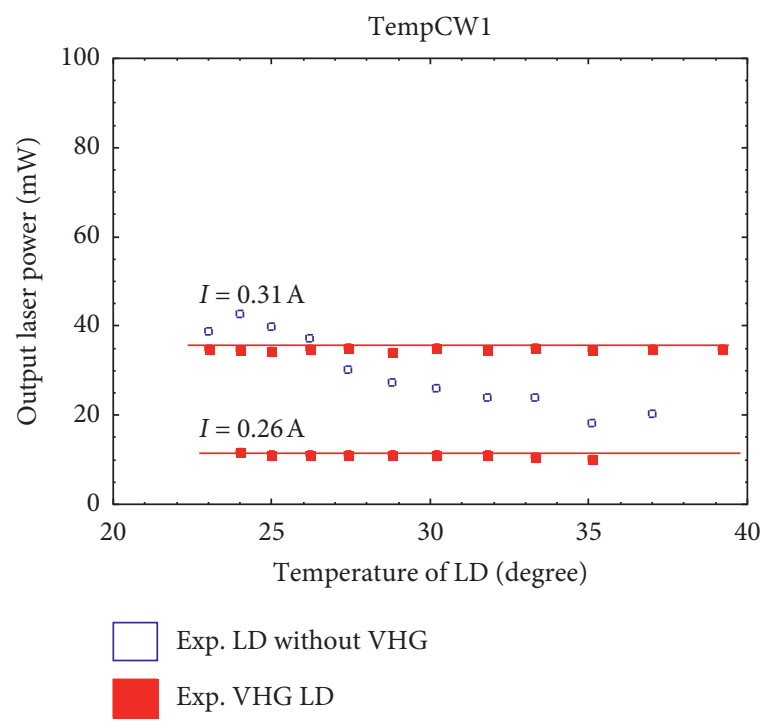

(a)

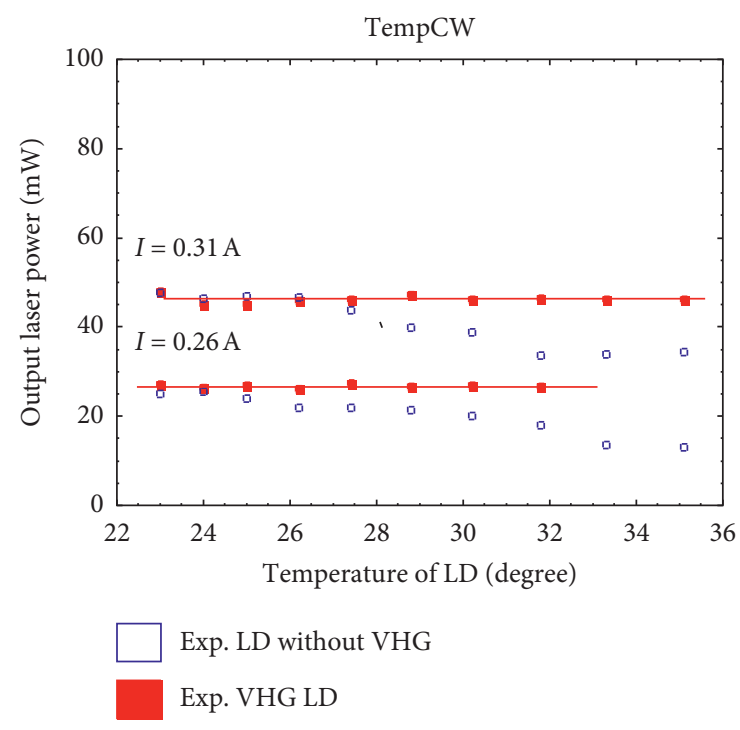

(b)

FIGURE 5: Stability of output laser power for LD temperature: (a) Nd:YAG and (b) Nd:YVO ${ }_{4}$

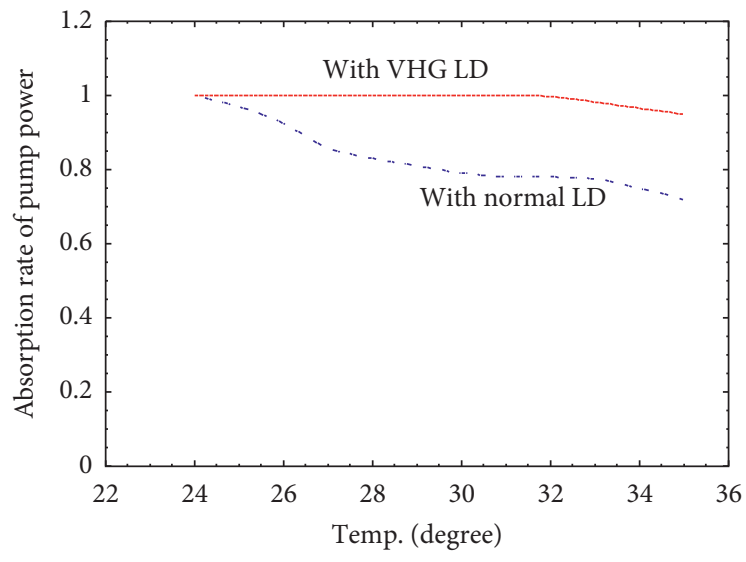

(a)

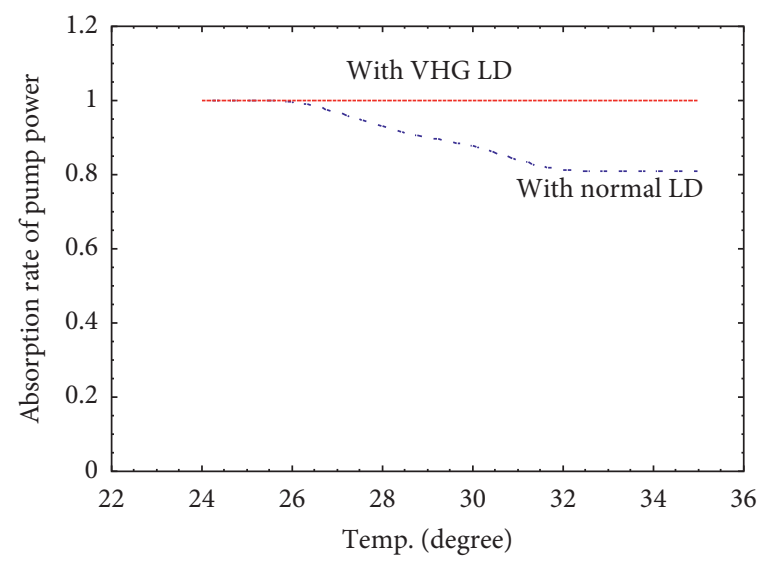

(b)

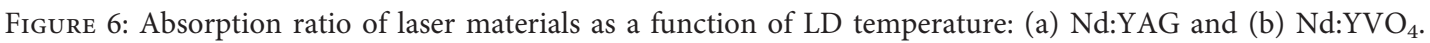




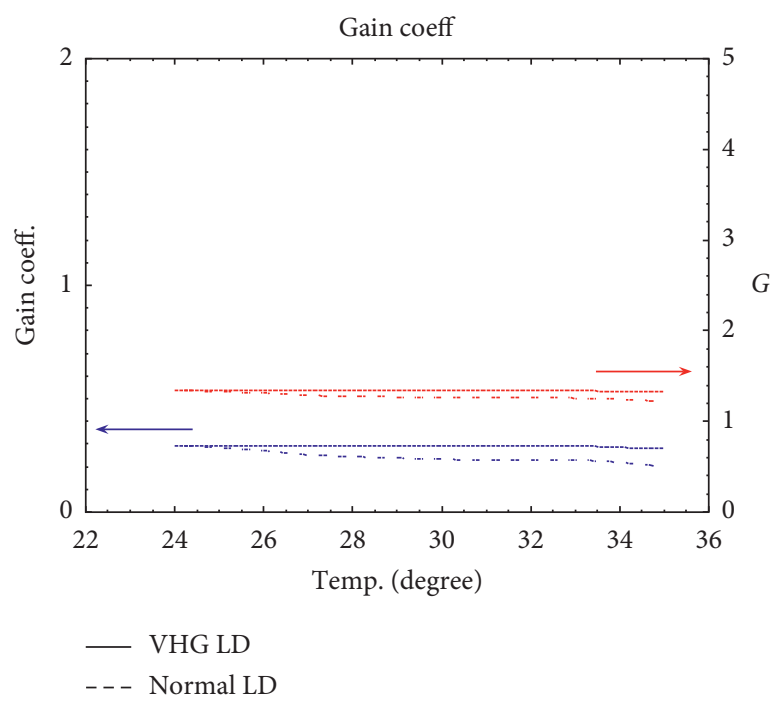

(a)

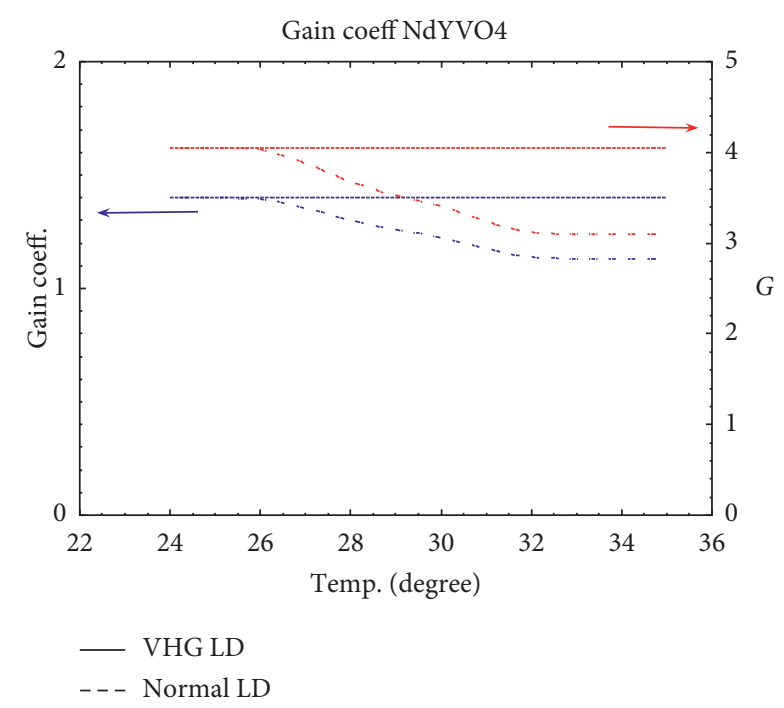

(b)

FIGURE 7: Calculated small signal gain coefficient and laser large gain: (a) Nd:YAG and (b) Nd:YVO

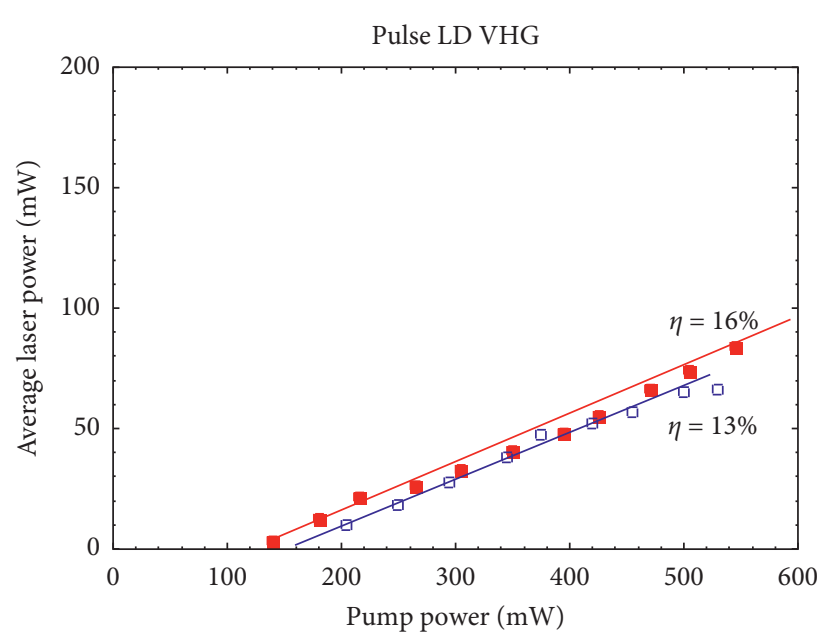

$\square$ Exp. LD without VHG

Exp. VHG LD

(a)

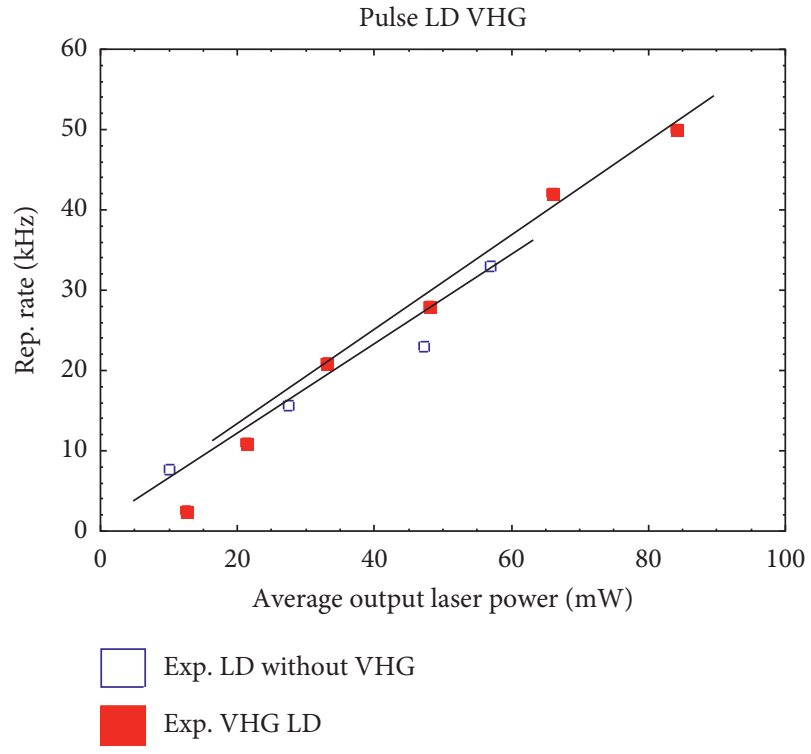

(b)

FIGURE 8: Nd:YAG laser under pulse operation: (a) average output laser power and (b) repetition rate.

13\%, as shown in Figure 8(a). The threshold was also $140 \mathrm{~mW}$ for this laser using the VHG LD, but the slope efficiency was $21 \%$ and maximum output conversion efficiency was 16\%, as shown in Figure 8(a). The conversion and slope efficiencies increased slightly by using the VHG LD.

The repetition rate varied from 7 to $36 \mathrm{kHz}$ for the $\mathrm{Nd}$ : YAG laser using the LD without VHG. It also varied from 2 to $60 \mathrm{kHz}$ when using the VHG LD, as shown in Figure 8(b). The pulse duration of this laser was $8 \mathrm{~ns}$, and the peak power was evaluated to be $225 \mathrm{~W}$.

For the laser using the $\mathrm{Nd}: \mathrm{YVO}_{4}$ laser using the LD without VHG, the threshold for the pump power was $160 \mathrm{~mW}$, slope efficiency was $50 \%$, and conversion efficiency at the maximum output was 30\%, as shown in Figure 9(a), and the maximum output laser power was $135 \mathrm{~mW}$. For this laser using the VHG LD, the threshold was $30 \mathrm{~mW}$, slope efficiency was $36 \%$, and maximum output conversion efficiency was $33 \%$, as shown in Figure 9(a). These results indicate that the conversion and slope efficiencies increased using the VHG LD. The obtained maximum output laser power was $180 \mathrm{~mW}$.

The repetition rate varied from 400 to $800 \mathrm{kHz}$ for the $\mathrm{Nd}: \mathrm{YVO}_{4}$ laser using the LD without VHG. It also varied from 400 to $900 \mathrm{kHz}$ for this laser using the VHG LD, as shown in Figure 9(b). The pulse duration of the laser was $28 \mathrm{~ns}$, and the peak power was evaluated to be $7.1 \mathrm{~W}$.

Experimental results for the stability of the output laser power with respect to LD temperature are shown in Figure 10. For the Nd:YAG laser using the LD without VHG, 


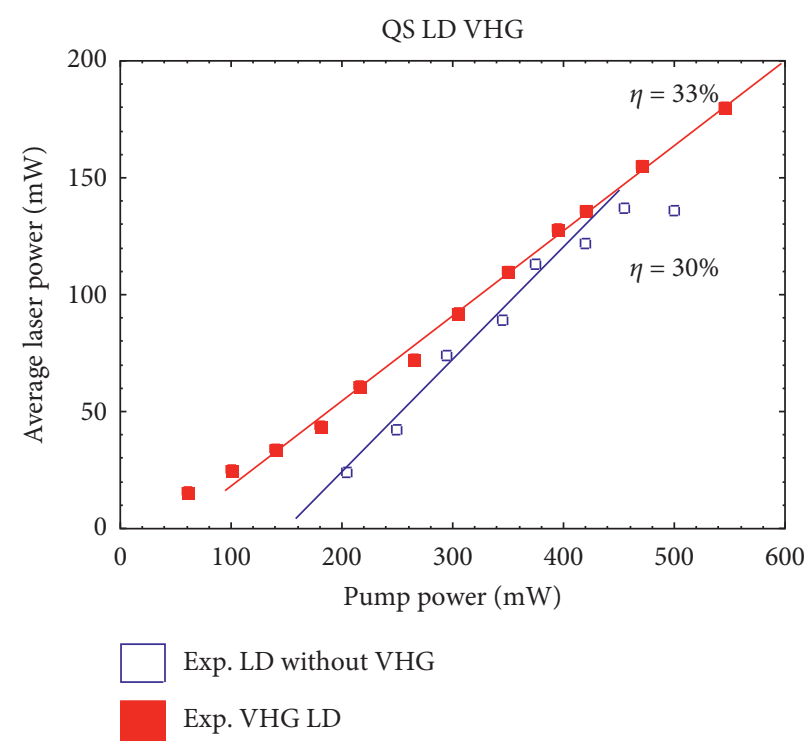

(a)

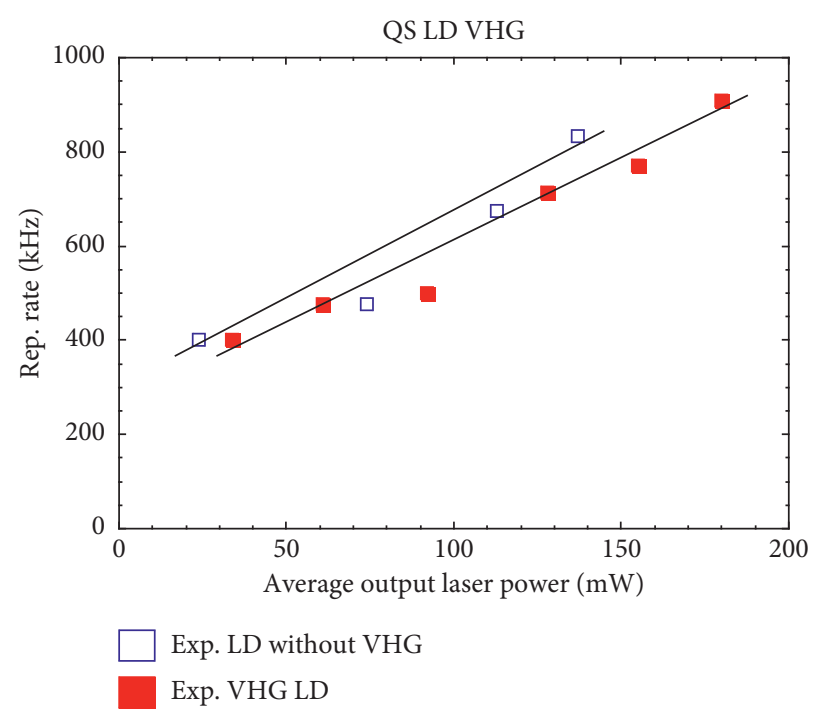

(b)

FIGURE 9: Nd: $\mathrm{YVO}_{4}$ laser under pulse operation: (a) average output laser power and (b) repetition rate.

the average output laser power was $16 \mathrm{~mW}$ at $25^{\circ} \mathrm{C}$, as shown in Figure 10(a), and the pump power was $200 \mathrm{~mW}$. When the temperature increased to $39^{\circ} \mathrm{C}$, this power decreased to $8 \mathrm{~mW}$. For this laser using the VHG LD, the average output laser power was $22 \mathrm{~mW}$ at $23^{\circ} \mathrm{C}$, as shown in Figure 10 (a), and the pump power was $210 \mathrm{~mW}$. When the temperature increased to $39^{\circ} \mathrm{C}$, this power was kept constant.

For the $\mathrm{Nd}: \mathrm{YVO}_{4}$ laser using the LD without VHG, the average output laser power was $20 \mathrm{~mW}$ at $23^{\circ} \mathrm{C}$, and the pump power was $200 \mathrm{~mW}$, as shown in Figure 10(b). The output laser power decreased to $15 \mathrm{~mW}$ when the temperature increased to $35^{\circ} \mathrm{C}$. For this laser using the VHG $\mathrm{LD}$, the average output laser power was $55 \mathrm{~mW}$ at $23^{\circ} \mathrm{C}$, and the pump power was $210 \mathrm{~mW}$. When the temperature increased to $35^{\circ} \mathrm{C}$, this power was kept constant.

These experimental results indicate that microchip lasers can be driven by simply cooling LDs to a temperature at which the LDs will not be damaged, even without a temperature controller. This enables us to simplify the device. Increasing the pump power of the LD light and obtaining a higher output laser power are for future work.

These microchip lasers are intended for operating up to around $40^{\circ} \mathrm{C}$. Because the heat generated in the laser material is below $0.5 \mathrm{~W}$, the temperature of the laser material is $10^{\circ} \mathrm{C}$ higher than the ambient temperature. Thus, the optical parameters of the laser materials should not change. However, when the output power of the LD light significantly increases, the laser material generates a large amount of heat and the optical parameters change. Thus, cooling the laser material and keeping the temperature constant are necessary.

In the case of CW laser emission, the slope efficiency depends on pump absorption efficiency, quantum efficiency, pump and resonator mode matching, quantum defect, output mirror transmission, and residual optical loss. In the case of laser materials with high quality, laser material and pump characteristics enable slope efficiency close to the quantum defect by selecting the resonator properly. An experimental result on the conversion efficiency in [3] is close to the quantum defect. However, in that study, the pump wavelengths were slightly longer than $808 \mathrm{~nm}$, which is slightly different from our experimental conditions. They obtained high conversion efficiency is $75 \%$. The maximum conversion efficiency at $808 \mathrm{~nm}$ pumping should be evaluated to be $71 \%$ considering the emission efficiency of $93 \%$ and quantum defect (excitation wavelength versus emission wavelength) of $76 \%$. In that study, CW laser emissions were reported. A short pulse laser was used for pumping with high intensity to increase the laser gain, and the LD output power was modulated by temporally controlling the current to increase pumping peak intensity with reducing thermal load. In experiments, the real emission efficiencies of $\mathrm{Nd}_{\mathrm{YVO}}$ and $\mathrm{Nd}$ :YAG are evaluated to be $100 \%[3,10]$.

In our study, the intensity of pump light was low, the laser gain was not high as fiber lasers, the ratio of mode utilization was low, the reflectance of the output mirror was high, and the extraction efficiency was low, so the conversion efficiency was limited to $60 \%$. However, the conversion efficiency will be higher than that of the standard $\mathrm{Nd}: \mathrm{YVO}_{4}$ microchip laser [2]. In our experiment, when the current was further controlled temporally and the LD output power was modulated to reduce the heat load, the pumping density increased, the laser gain increased, and the output mirror reflectance decreased. The conversion efficiency should approach $71 \%$ under quasi-CW oscillation, and higher output power should be obtained. Also, in pulse mode, conversion efficiency should improve and high peak power should be obtained. 


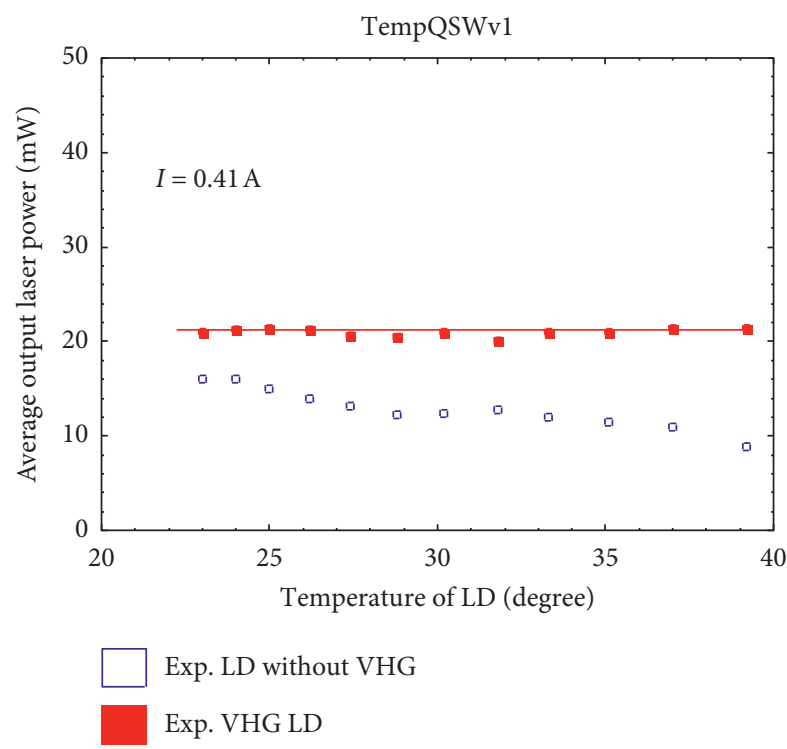

(a)

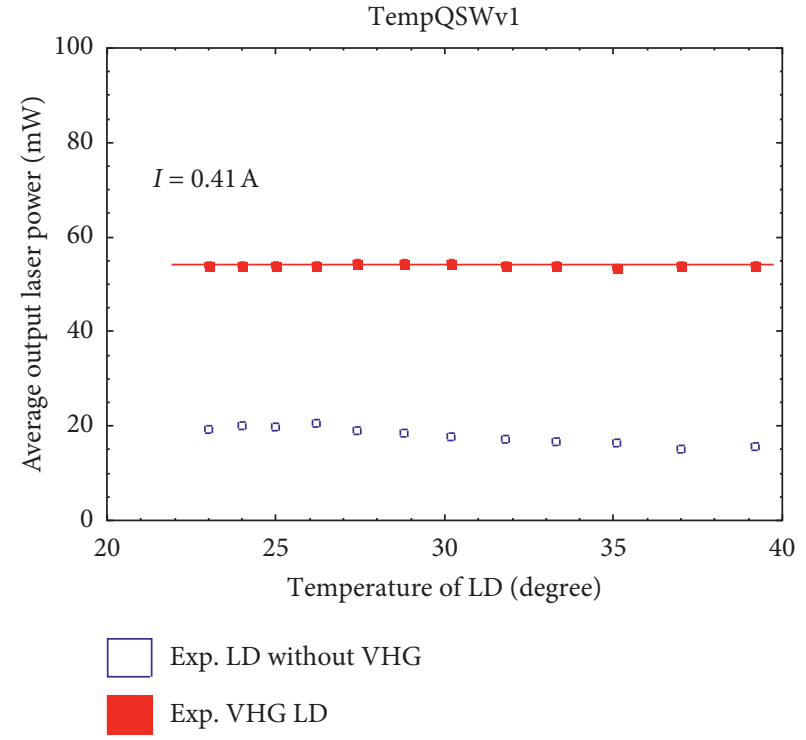

(b)

Figure 10: Stability of laser power for LD temperature: (a) Nd:YAG and (b) Nd:YVO

\section{Conclusion}

We developed end-pumped microchip Nd:YAG and $\mathrm{Nd}$ : $\mathrm{YVO}_{4}$ lasers pumped using a VHG LD as a pumping LD. We experimentally obtained high optical-optical conversion efficiency of close to $60 \%$ for the $\mathrm{Nd}: \mathrm{YVO}_{4}$ laser during $\mathrm{CW}$ operation. The output laser power is stable for the LD temperature of close to $40^{\circ} \mathrm{C}$ when using the VHG LD. The output laser power was calculated numerically to compare the experimental results. These experimental results will lead to the development of end-pump microchip lasers that do not require a temperature controller. The output laser power of the $\mathrm{Nd}: \mathrm{YVO}_{4}$ laser using the VHG LD was more than twice as high as that using the LD without VHG.

\section{Data Availability}

No data were used to support this study.

\section{Conflicts of Interest}

The authors declare no conflicts of interest.

\section{References}

[1] E. Molva, "Microchip lasers and their applications in optical microsystems," Optical Materials, vol. 11, no. 2-3, pp. 289299, 1999.

[2] R. A. Fields, M. Birnbaum, and C. L. Fincher, "Highly efficient $\mathrm{Nd}: \mathrm{YVO}_{4}$ diode-laser end-pumped laser," Applied Physics Letters, vol. 51, no. 23, pp. 1885-1886, 1987.

[3] Y. Sato, T. Taira, N. Pavel, and V. Lupei, "Laser operation with near quantum-defect slope efficiency in $\mathrm{Nd}_{\mathrm{YVO}} \mathrm{YV}_{4}$ under direct pumping into the emitting level," Applied Physics Letters, vol. 82, no. 6, pp. 844-846, 2003.
[4] A. Kausas and T. Taira, "Giant-pulse Nd:YVO ${ }_{4}$ microchip laser with MW-level peak power by emission cross-sectional control," Optics Express, vol. 24, no. 4, pp. 3137-3149, 2016.

[5] J. J. Zayhowski and A. Mooradian, "Frequency-modulated Nd:YAG microchip lasers," Optics Letters, vol. 14, no. 12, pp. 618-620, 1989.

[6] G. A. Kumar, J. Lu, A. A. Kaminskii et al., "Spectroscopic and stimulated emission Characteristics of $\mathrm{Nd} /$ sup $3+/$ in transparent YAG ceramics," IEEE Journal of Quantum Electronics, vol. 40, no. 6, pp. 747-758, 2004.

[7] J. J. Zayhowski and A. Mooradian, "Single-frequency microchip Nd lasers," Optics Letters, vol. 14, no. 1, pp. 24-26, 1989.

[8] T. Dascalu, N. Pavel, and T. Taira, "90 W continuous-wave diode edge-pumped microchip composite $\mathrm{Yb}_{\mathrm{Y}} \mathrm{Y}_{3} \mathrm{Al}_{5} \mathrm{O}_{12}$ laser," Applied Physics Letters, vol. 83, no. 20, pp. 4086-4088, 2003.

[9] J. J. Zayhowski and C. Dill, "Diode-pumped passively Q-switched picosecond microchip lasers," Optics Letters, vol. 19, no. 18, pp. 1427-1429, 1994.

[10] V. Lupei, N. Pavel, and T. Taira, "Basic enhancement of the overall optical efficiency of intracavity frequency-doubling devices for the $1 \mu \mathrm{m}$ continuous-wave $\mathrm{Nd}: \mathrm{Y}_{3} \mathrm{Al}_{5} \mathrm{O}_{12}$ laser emission," Applied Physics Letters, vol. 83, no. 18, pp. 3653-3655, 2003.

[11] H. Sakai, H. Kan, and T. Taira, " $>1$ MW peak power singlemode high-brightness passively Q-switched $\mathrm{Nd}^{3+}$ :YAG microchip laser," Optics Express, vol. 16, no. 24, pp. 19891-19899, 2008.

[12] N. Pavel, J. Saikawa, S. Kurimura, and T. Taira, "High average power diode end-pumped composite Nd:YAG laser passively Q-switched by $\mathrm{Cr}^{4+}$ :YAG saturable absorber," Japanese Journal of Applied Physics, vol. 40, no. Part 1, No. 3A, pp. 1253-1259, 2001.

[13] N. Pavel, M. Tsunekane, and T. Taira, "Composite, all-ceramics, high-peak power $\mathrm{Nd}: \mathrm{YAG} / \mathrm{Cr}^{4+}: \mathrm{YAG}$ monolithic micro-laser with multiple-beam output for engine ignition," Optics Express, vol. 19, no. 10, pp. 9378-9384, 2011.

[14] O. Sandu, G. Salamu, N. Pavel et al., "High-peak power, passively Q-switched, composite, all-polycrystalline ceramic 
Nd:YAG/Cr ${ }^{4+}$ :YAG lasers," Quantum Electronics, vol. 42, no. 3, pp. 211-215, 2012.

[15] A. V. Podlipensky, K. V. Yumashev, N. V. Kuleshov, H. M. Kretschmann, and G. Huber, "Passive Q-switching of $1.44 \mu \mathrm{m}$ and $1.34 \mu \mathrm{m}$ diode-pumped Nd:YAG lasers with a V: YAG saturable absorber," Applied Physics B: Lasers and Optics, vol. 76, no. 3, pp. 245-247, 2003.

[16] V. Lupei, N. Pavel, Y. Sato, and T. Taira, "Highly efficient 1063-nm continuous-wave laser emission in $\mathrm{Nd}: \mathrm{GdVO}_{4}$," Optics Letters, vol. 28, no. 23, pp. 2366-2368, 2003.

[17] W. Koechner, Solid-State Laser Engineering, Springer, New York, NY, USA, Chapter 2, 6th edition, 2006.

[18] Y. Kotaki, M. Matsuda, H. Ishikawa, and H. Imai, "Tunable DBR laser with wide tuning range," Electronics Letters, vol. 24, no. 8, pp. 503-505, 1988.

[19] H. Takaaki, M. Minoru, S. Masayuki, and H. Haruo, "Fabrication and characteristics of $\mathrm{GaAs} / \mathrm{AlGaAs}$ tunable laser diodes with DBR and phase-control sections integrated by compositional disordering of a quantum well," IEEE Journal of Quantum Electronics, vol. 27, no. 6, pp. 1609-1615, 1991.

[20] S.-T. Dai, J.-H. Huang, H.-Z. Huang et al., "Design and performance of a composite Tm:YAG laser pumped by VBGstabilized narrow-band laser diode," Chinese Physics B, vol. 26, no. 7, Article ID 074211, 2017.

[21] V. Jambunathan, L. Horackova, P. Navratil, A. Lucianetti, and T. Mocek, "Cryogenic Yb:YAG laser pumped by VBG-stabilized narrowband laser diode at $969 \mathrm{~nm}$," IEEE Photonics Technology Letters, vol. 28, no. 12, pp. 1328-1331, 2016.

[22] A. Yariv and P. Yeh, "Photonics: optical electronics in modern communication," The Oxford Series in Electrical and Computer Engineering, Oxford University Press, 6th edition, 2007. 